Vol. (7) 1: pp. 24-32

\title{
- SOPORTE Institucional PARA LA Investigación Científica Y Tecnológica en el Perú
}

\section{RESUMEN}

El estudio propone el fortalecimiento Institucional de las organizaciones que fomentan la Investigación científica y tecnológica, a fin de establecer una política de Estado sobre el desarrollo de la C y T, que dinamice la inversión y facilite el crecimiento de la productividad en empresas, para garantizar en el mediano y largo plazo la competitividad de los bienes y servicios, y mejorar las condiciones de vida de la población peruana.

Palabras Claves: Investigación. Ciencia y tecnología. Soporte institucional.

INSTITUTIONAL SUPPORT FOR THE Technological and Scientifical Research in Peru ABSTRACT

This work proposes an institutional strengthening of organizations encouraging technological and scientifical research, with the purpose of stating a government policy on Science and Technology development, one able to boost investment and provide with productivity growing in enterprises, in order to guarantee goods and services competitiveness, and improve peruvian population living conditions both in the long and short run.

Key Words: Research. Science and technology. Institutional support.

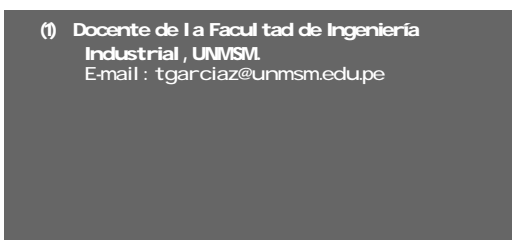

\section{INTRODUCCIÓN}

La presente investigación expone los diferentes enfoques y conocimiento sobre la situación actual de las diversas organizaciones dedicadas a la investigación científica y tecnológica en el Perú, las mismas que se encuentran bajo la administración y gestión de diversos ministerios, universidades y ONGs, y cada cual desarrolla investigación científica y tecnológica según su saber y entender, sin ninguna planificación ni orientación de lo que el país necesita, esto hace que las investigaciones sigan una suerte de desorden dentro de un país tan rico en recursos naturales y humanos, pero tan frágil en la definición de prioridades reales que conlleven a no tener claro lo que necesita nuestro país en Investigación científica y tecnológica, a nivel nacional, regional y local.

Señala las limitaciones técnico-normativas de CONCYTEC que limitan el desarrollo de un trabajo sistémico y a ello se suma la falta de voluntad política para establecer un ordenamiento institucional que promueva una política nacional científico tecnológica, que lo vincule con las universidades, la comunidad científica, el empresariado y la sociedad civil mirando hacia las potencialidades del país; mantienen además la insuficiencia y el enorme atraso en las formas y mecanismos de financiamiento público y privado, así como en un inadecuado estímulo de incentivos a la investigación lo cual deriva en desaprovechamiento y fuga de talentos y en incipiente generación de conocimientos e innovación.

\section{OBJ ETIVOS}

Los objetivos que se han propuesto en el presente estudio se mencionan a continuación:

1. Reordenar las instituciones involucradas en el campo de la Ciencia y Tecnología dentro del marco institucional de la Reforma del Estado, que propicie el fortalecimiento Institucional bajo el enfoque sistémico para que promueva la formulación de una política de Estado coherente y un PLAN NACIONAL DE CIENCIA Y TECNOLOGÍA debidamente coordinado a nivel nacional, regional y local, orientando así las políticas, planes y programas en función de las necesidades del país en el corto, mediano y largo plazo para el mejor aprovechamiento de sus ventajas y recursos.

2. Promover el desarrollo científico y tecnológico para facilitar el crecimiento de la productividad en empresas y organizaciones, con la finalidad de poder garantizar en el mediano y largo plazo la competitividad de los bienes y servicios de las MYPEs. 
3. Promover la investigación científica y tecnológica proponiendo incentivos y estímulos a los investigadores, e inventores.

4. Contribuir con el Gobierno actual, en la organización de una administración y gestión moderna de sus capacidades en el marco de la Reforma del Estado.

\section{FUNDAMENTACIÓN DEL PROBLEMA}

La duplicidad de funciones ante la carencia de una institución rectora y el marco legal que defina las funciones de las diferentes instituciones involucradas en el desarrollo de la ciencia y tecnología, no permiten promover en forma armónica y conjunta el desarrollo de la investigación científica y tecnológica en el ámbito nacional.

Esta situación se hace sentir desde que desactivaron el Instituto Nacional de Planificación y hasta el momento el país es un barco a la deriva, pues la ausencia de una percepción generalizada de la importancia de la ciencia y la tecnología para el desarrollo y para la solución de problemas de orden social y productivo se percibe en todo el territorio nacional y por que no a nivel internacional (por las fuentes cooperantes).

A todo esto, se suma el olvido o escasa atención del Estado, que ha descuidado su importante rol articulador, organizador y catalizador de los esfuerzos nacionales en materia de ciencia y tecnología.

El Estado no genera las condiciones propicias al desarrollo científico - tecnológico ni permite el despliegue de las potencialidades del país, dando lugar no solo al estancamiento sino la exclusión, por la ampliación desmedida de la brecha de nuestro país con respecto al resto del mundo.

\section{IMPORTANCIA Y JUSTIFICACIÓN DE LA INVESTIGACIÓN}

En una época caracterizada como la sociedad del conocimiento, donde la fuente de creación de riqueza es el hombre capacitado de manera continua, se debe estar en condiciones de reconocer y darle prioridad a la investigación científica y tecnológica para el desarrollo y la competitividad, caso contrario estaremos negando a las futuras generaciones la base fundamental para poder desarrollarse y escapar de la pobreza.

Actualmente en nuestro País, no obstante que existe una Institución orientada a la investigación científi- ca y tecnológica, ésta se encuentra supeditada al Ministerio de Educación, lo que les impide un trabajo coherente y con poder de decisión para normar la política integral de ciencia y tecnología.

Mayormente los esfuerzos realizados no tienen resultados concretos, debido a la ausencia de objetivos claros y precisos sobre el alcance que debe tener el desarrollo de la ciencia y tecnología dentro del marco de un ordenamiento institucional para el desarrollo del País.

El hecho mismo de conocer que las diversas instituciones dedicadas a la investigación se encuentran bajo la administración y gestión de diversos ministerios y universidades y cada cual desarrolla investigación científica y tecnológica según su saber y entender, sin ninguna planificación ni orientación de lo que el País necesita.

En este contexto, las investigaciones siguen una suerte de desorden dentro de un País tan rico en recursos naturales y humanos, pero tan frágil en la definición de prioridades reales que conlleven a no tener claro lo que necesita nuestro país en la materia, a nivel nacional, regional y local.

Asimismo, la falta de un reordenamiento institucional que integre en un solo organismo a todas las instituciones vinculadas con la actividad científica y tecnológica impiden el establecimiento de lineamientos y políticas nacionales en materia de ciencia, tecnología e innovación, así como para la estimación de los recursos necesarios para su ejecución, hace que se justifique la elaboración de esta investigación.

\section{PLANTEAMIENTO DEL PROBLEMA}

La principal interrogante planteada fue la siguiente:

¿Será el reordenamiento institucional de la actividad científica y tecnológica un mecanismo que permita organizar y priorizar las necesidades mas sentidas de nuestra población a nivel nacional regional y local, que conlleve a identificar el tipo o tipos de investigación que requiere el país para el desarrollo socioeconómico y la promoción de la inversión?

\section{DISEÑO DE LA METODOLOGÍA}

La población universo son todas las instituciones dedicadas a la investigación científica y tecnológica del Perú, incluidas lasuniversidades. Por la naturaleza de la población, se utilizó un muestreo denominado discrecional, basado en el criterio de los investigado- 
res por la experiencia sobre los elementos que son elegidos para el desarrollo del estudio.

Se propuso como primera hipótesis que a mayor organización del sistema administrativo de ciencia y tecnología, mayores posibilidades de priorizar los proyectos de investigación científica y tecnológica que promuevan el desarrollo del País.

En lo que respecta a las técnicas de recopilación de la información utilizadas fueron:

a. Recopilación de las leyes orgánicas, que norman el funcionamiento de las Instituciones dedicadas al la investigación científica y tecnológica.

b. Recopilación de las leyes orgánicas que norman el funcionamiento de los Gobiernos Regionales y Locales.

c. Recopilación de la información de documentos de gestión de cada uno de ellos, y una encuesta a los funcionarios, sobre su opinión con relación al funcionamiento de sus respectivas instituciones y de los investigadores en general.

d. Testimonios de los funcionarios que trabajan en regiones y municipios sobre la apreciación de los Gobiernos Regionales y Gobiernos Locales con relación al funcionamiento de la ciencia y tecnología.

e. Libros relacionados con la reforma del Estado y la ciencia y tecnología.

f. Pronunciamientos diversos y estudios de la Comisión de Descentralización y Reforma del Estado del Congreso de la República.

g. Testimonios de los docentes de diversas Universidades Públicas y Privadas en relación a como de desarrolla la investigación científica y tecnológica dentro de su universidad.

De las técnicas estadísticas, se está empleando el análisis descriptivo, explicativo y de comprensión a través del procesamiento de la información.

\section{PRIMEROS RESULTADOS E IMPLICANCIAS}

La presente investigación está permitiendo demostrar que las entidades vinculadas a la investigación científica y tecnológica en el desorden y dispersión en la que se encuentran, no nos han traído a la fecha soluciones a las necesidades mas sentidas de nuestra población, por ello la pretensión de proponer como punto importante de la investigación la organización de las entidades vinculadas a la investigación científica y tecnológica bajo un enfoque sistémico, ya que esto supone un esfuerzo técnico para la priorización del tipo de investigación que requiere el país, la planificación del presupuesto tanto público como priva- do en materia de investigación científica y tecnológica, encaminado al alcance de los objetivos estratégicos de desarrollo del Perú, en función de sus recursos existentes, mediante el diseño del desarrollo organizacional que conlleve a orientar los instrumentos financieros y de las modalidades de participación, capaces de estimular las actividades de investigación científica y tecnológica, dentro del contexto de la escena internacional y el esfuerzo de la cooperación y coordinación entre las organizaciones gestoras de la administración central del Estado, los Gobiernos Regionales y Locales.

En la medida que se logre esta organización del Sistema del Consejo de Ciencia y Tecnología u otra similar, se podrá elaborar un Plan Nacional de largo plazo, estructurado en áreas prioritarias, donde se podrá incluir el financiamiento correspondiente, así como mecanismos de seguimiento, evaluación y cumplimiento de los proyectos o programas y medir los impactos que se estuvieran produciendo.

Los conceptos pasados de eficiencia para la organización, los modelos de gerencia, la concentración de las actividades administrativas, económicas, financieras y de la población en Lima Metropolitana del pasado y del presente, deberán servir para tener una idea de lo que no se debe repetir ahora y en el futuro, puesto que con la introducción de la informática, las telecomunicaciones y la globalización de la economía, han revolucionado todas las actividades que debemos revisar para entrar a la modernidad de la administración y gestión pública.

La multiplicación de las entidades del estado dedicadas a la investigación, ciencia y tecnología descoordinados entre sí y con una Institución tal como CONCYTEC, dependiente del Ministerio de Educación, no tiene el suficiente poder y/o autonomía para trabajar bajo el contexto que se está proponiendo.

De la revisión de la misión, visión, planes y funciones de CONCYTEC, de igual forma que INIA, IPEN, INGEMMET, INCITEMI, INGEOMIN, INAIT, INICTEL, IIAP, ITP, CONIDA, CONACS, IAP, IMARPE, IGN, IGP, INEI, INRENA, INS, SENAMHI, SENASA, entre otros, que son nuestras principales fuentes de información para demostrar la tesis propuesta, así como la información de las Universidades Públicas y Privadas en materia de investigación, y los testimonios recogidos, ha quedado claro la falta de orden y la duplicidad de funciones en materia de investigación científica y tecnológica en el Perú.

Los indicadores obtenidos por el mismo Consejo de Ciencia y Tecnología están dando una idea de lo mal que está el País en este aspecto; como es el caso 
Cu a d r o 1. Patentes de invención y Tasa de autosuficiencia Perú versus Extranjero (1993-2002)

\begin{tabular}{|c|c|c|c|c|c|c|c|c|c|c|}
\hline \multirow[b]{2}{*}{ INDICADORES } & \multicolumn{10}{|c|}{ AÑO } \\
\hline & 1993 & 1994 & 1995 & 1996 & 1997 & 1998 & 1999 & 2000 & 2001 & 2002 \\
\hline \multicolumn{11}{|l|}{ Patentes de invención otorgadas por origen } \\
\hline \multirow{2}{*}{$\begin{array}{l}\text { Nacionales } \\
\text { Extranieras }\end{array}$} & 10 & 15 & 9 & 7 & 7 & & 5 & 9 & 14 & $\underline{22}$ \\
\hline & 104 & 221 & 267 & 174 & 173 & 132 & 266 & 299 & 523 & 528 \\
\hline \multicolumn{11}{|l|}{ Tasa de autosuficiencia } \\
\hline Patentes solic. por residentes / Total solicitudes & 0.10 & 0.07 & 0.04 & 0.09 & 0.06 & 0.04 & 0.05 & 0.04 & 0.04 & 0.04 \\
\hline \multicolumn{11}{|l|}{ Tasa de dependencia } \\
\hline Patentes solic. por no residentes / Patentes solic. por & 8.67 & 13.1 & 22.8 & 10.8 & 15.30 & 24.9 & 19.3 & 25.98 & 26.2 & 26.6 \\
\hline
\end{tabular}

Fuente: Perú ante la sociedad del conocimiento, indicadores de ciencia y tecnología e innovación. 1960-2002

del número reducido de investigadores y de profesores universitarios con la formación y grados académicos adecuados y el número de patentes por inventos registrados anualmente. A este problema se añade la escasa o nula comunicación entre la investigación y las actividades sociales y productivas que impide el uso de los conocimientos generados. El no estar acreditadas la mayoría de Universidades Públicas y Privadas, así como las diferentes carreras profesionales, son otro indicador de lo mal que andan nuestras fuentes del saber y del conocimiento.

En cuanto a los indicadores de resultados referidos a patentes que permiten estimar el grado de autosuficiencia o de dependencia en la generación de conocimientos, en la medida en que dichas patentes protegen conocimientos con potencial interés económico hacen mas fuerte un país, caso contrario estamos demostrando nuestra gran dependencia científica y tecnológica ante la incapacidad inventiva.

En cualquier país las patentes se otorgan a residentes y no residentes; en el caso del Perú, si bien es cierto estas se han incrementado hasta alcanzar en el año 2002, 528 patentes a no residentes y 22 patentes a residentes, que arroja una tasa de autosuficiencia de 0,04. Dicha tasa crece cuando es mayor el número de patentes otorgadas a los residentes. La tasa de autosuficiencia en el Perú es muy baja, lo que refleja el escaso dinamismo tecnológico y consecuentemente la limitada actividad inventiva del país. Lo expuesto se evidencia con mayor claridad cuando se hace un análisis comparativo entre países de América Latina, en este caso la tasa de dependencia alcanza al 26,6\% al mismo año.

Como se puede apreciar en el Cuadro 2 y la Figura 1 , los recursos destinados a la inversión en investigación científica y desarrollo experimental son exiguos. Asimismo, se muestra la perfomance de los diferentes países en comparación con el Perú y se deduce rápidamente porque se está en dicha situación. Es decir, es casi cero en esta materia y por ende se siente el atraso y la dependencia.

EI Perú invierte el 0,11\% del su PBI en Investigación y Desarrollo para el año 2002, incluyendo el gasto público y de universidades y empresas privadas, mientras que Canadá invierte el 1,93\% y Estados Unidos 2,76\%, o se observa al Brasil con el $1.05 \%$ del PBI apreciando su avance en el desarrollo entre los países de América Latina.

Cabe mencionar que los recursos financieros destinados a las actividades de ciencia y tecnología, no se traducen en mejoras del potencial científico y tecnológico del país. En la actualidad existen registradas 1121 instituciones y dependencias vinculadas a actividades científicas y tecnológicas, entre ellas 1088 escuelas académico -profesionales que corresponden a facultades de 79 universidades y 33 instituciones públicas vinculadas a la ciencia y tecnología. El accionar del conjunto de estas instituciones está caracterizado por el esfuerzo aislado y disperso que, lejos de complementarse en el logro de aportes significativos y de calidad, genera duplicidad y mal uso de los recursos. Es de observar en el Figura 2 y el Cuadro 3 la distribución del gasto público en actividades de ciencia y Tecnología por las instituciones públicas, siendo estas muy reducidas. Asimismo se aprecia la incongruencia y disparidad de las actividades que se desarrollan.

El $57.1 \%$ de nuestros estudiantes universitarios se orientan hacia las ciencias sociales, y si a esto le sumáramos humanidades llegaríamos a un total de $60.6 \%$, en tanto para las Ingenierías solo se dedican el $18 \%$ de nuestros estudiantes, lo cual proporciona un dato contundente con respecto a la poca importancia que desde la formación profesional arrastra nuestra nación.

Es evidente la falta de especialización, de concentración y optimización de esfuerzos en áreas más especí- 
Cu a d r o 2. Gastos en Ciencia y Tecnología en relación al PBI

\begin{tabular}{|c|c|c|c|c|c|c|c|c|c|c|c|c|c|c|}
\hline País & & 1990 & 1991 & 1992 & 1993 & 1994 & 1995 & 1996 & 1997 & 1998 & 1999 & 2000 & 2001 & 2002 \\
\hline \multirow{2}{*}{ Argentina } & ACT & $33.00 \%$ & $0.34 \%$ & $0.38 \%$ & $0.43 \%$ & $0.44 \%$ & $0.49 \%$ & $50 \%$ & $0.50 \%$ & $0.50 \%$ & $0.52 \%$ & $0.50 \%$ & $0.48 \%$ & $0.44 \%$ \\
\hline & I+D & & & & & & & $0.42 \%$ & $0.42 \%$ & $0.41 \%$ & $0.45 \%$ & $0.44 \%$ & $0.42 \%$ & $0.39 \%$ \\
\hline \multirow{2}{*}{ Bolivia } & ACT & & & & & & & & $0.58 \%$ & $0.54 \%$ & $0.55 \%$ & $0.54 \%$ & $0.52 \%$ & $0.51 \%$ \\
\hline & I+D & & & $0.37 \%$ & $0.40 \%$ & $0.40 \%$ & $0.36 \%$ & $0.33 \%$ & $0.32 \%$ & $0.29 \%$ & $0.29 \%$ & $0.28 \%$ & $0.27 \%$ & $0.26 \%$ \\
\hline \multirow{2}{*}{ Brasil } & ACT & $1.59 \%$ & $1.81 \%$ & $1.64 \%$ & $1.79 \%$ & $1.68 \%$ & $1.40 \%$ & $1.22 \%$ & & & $1.33 \%$ & & & \\
\hline & I+D & $0.76 \%$ & $0.89 \%$ & $0.76 \%$ & $0.91 \%$ & $0.92 \%$ & $0.87 \%$ & $0.77 \%$ & & & $0.86 \%$ & $1.04 \%$ & & \\
\hline Canadá & I+D & $1.51 \%$ & $1.57 \%$ & $1.62 \%$ & $1.68 \%$ & $1.73 \%$ & $1.70 \%$ & $1.65 \%$ & $1.66 \%$ & $1.76 \%$ & $1.79 \%$ & $1.89 \%$ & $2.00 \%$ & $1.88 \%$ \\
\hline Chile & I+D & $0.51 \%$ & $0.53 \%$ & $0.58 \%$ & $0.63 \%$ & $0.62 \%$ & $0.62 \%$ & $0.58 \%$ & $0.54 \%$ & $0.54 \%$ & $0.55 \%$ & $0.56 \%$ & $0.57 \%$ & \\
\hline \multirow{2}{*}{ Colombia } & ACT & & & & & & $0.55 \%$ & $0.63 \%$ & $0.58 \%$ & $0.38 \%$ & $0.41 \%$ & $0.39 \%$ & $0.31 \%$ & $0.22 \%$ \\
\hline & I+D & & & & & & $0.29 \%$ & $0.34 \%$ & $0.30 \%$ & $0.21 \%$ & $0.20 \%$ & $0.18 \%$ & $0.17 \%$ & $0.10 \%$ \\
\hline \multirow{2}{*}{ Costa Rica } & ACT & $0.73 \%$ & $0.81 \%$ & $0.97 \%$ & $1.11 \%$ & $0.97 \%$ & $0.98 \%$ & $1.35 \%$ & $1.28 \%$ & $1.17 \%$ & $0.83 \%$ & $0.93 \%$ & & \\
\hline & I+D & & & & & & & $0.30 \%$ & $0.29 \%$ & $0.26 \%$ & $0.33 \%$ & $0.39 \%$ & & \\
\hline \multirow{2}{*}{ Cuba } & ACT & $1.09 \%$ & $1.16 \%$ & $1.66 \%$ & $1.32 \%$ & $0.98 \%$ & $0.87 \%$ & $0.78 \%$ & $0.82 \%$ & $0.93 \%$ & $1.01 \%$ & $1.03 \%$ & $1.12 \%$ & $1.10 \%$ \\
\hline & I+D & $0.70 \%$ & $0.68 \%$ & $1.13 \%$ & $0.78 \%$ & $0.55 \%$ & $0.47 \%$ & $0.38 \%$ & $0.43 \%$ & $0.54 \%$ & $0.50 \%$ & $0.52 \%$ & $0.61 \%$ & $0.62 \%$ \\
\hline \multirow{2}{*}{ Ecuador } & ACT & & & & & & & $0.20 \%$ & $0.26 \%$ & $0.27 \%$ & & & & \\
\hline & I+D & & & & & & $0.08 \%$ & $0.10 \%$ & $0.09 \%$ & $0.09 \%$ & & & & \\
\hline \multirow{2}{*}{ EI Salvador } & ACT & & & & & $0.34 \%$ & $0.35 \%$ & $0.35 \%$ & $0.35 \%$ & $0.95 \%$ & & & & \\
\hline & I+D & & & & & & & & & $0.09 \%$ & & & & \\
\hline España & I+D & $0.85 \%$ & $0.87 \%$ & $0.91 \%$ & $0.91 \%$ & $0.85 \%$ & $0.81 \%$ & $0.83 \%$ & $0.82 \%$ & $0.89 \%$ & $0.88 \%$ & $0.94 \%$ & $0.95 \%$ & $1.03 \%$ \\
\hline \begin{tabular}{|l|}
$\begin{array}{l}\text { Estados } \\
\text { Unidos }\end{array}$ \\
\end{tabular} & $\mathrm{I}+\mathrm{D}$ & $2.62 \%$ & $2.69 \%$ & $2.61 \%$ & $2.49 \%$ & $2.39 \%$ & $2.48 \%$ & $2.52 \%$ & $2.55 \%$ & $2.59 \%$ & $2.63 \%$ & $2.70 \%$ & $2.71 \%$ & $2.64 \%$ \\
\hline \multirow{2}{*}{ México } & ACT & $0.28 \%$ & $0.33 \%$ & $0.32 \%$ & $0.37 \%$ & $0.41 \%$ & $0.35 \%$ & $0.35 \%$ & $0.42 \%$ & $0.46 \%$ & $0.41 \%$ & $0.42 \%$ & $0.41 \%$ & $0.41 \%$ \\
\hline & I+D & & & & $0.22 \%$ & $0.29 \%$ & $0.31 \%$ & $0.31 \%$ & $0.34 \%$ & $0.38 \%$ & $0.43 \%$ & $0.37 \%$ & $0.39 \%$ & \\
\hline \multirow{2}{*}{ Panamá } & ACT & $0.63 \%$ & $0.67 \%$ & $0.63 \%$ & $0.71 \%$ & $0.72 \%$ & $0.76 \%$ & $0.85 \%$ & $0.92 \%$ & $0.89 \%$ & $0.94 \%$ & $0.91 \%$ & $1.03 \%$ & \\
\hline & I+D & $0.38 \%$ & $0.38 \%$ & $0.34 \%$ & $0.36 \%$ & $0.37 \%$ & $0.38 \%$ & $0.38 \%$ & $0.37 \%$ & $0.34 \%$ & $0.35 \%$ & $0.40 \%$ & $0.40 \%$ & \\
\hline \multirow{2}{*}{ Perú } & ACT & & & & $0.76 \%$ & $0.64 \%$ & $0.88 \%$ & $0.91 \%$ & $0.99 \%$ & $1.11 \%$ & $1.25 \%$ & $1.29 \%$ & $1.44 \%$ & $1.40 \%$ \\
\hline & I+D & & & & & & & & $0.08 \%$ & $0.10 \%$ & $0.10 \%$ & $0.11 \%$ & $0.11 \%$ & $0.10 \%$ \\
\hline \multirow{2}{*}{$\begin{array}{l}\text { Trinidad y } \\
\text { Tobago }\end{array}$} & ACT & & & & & & & $0.28 \%$ & $0.30 \%$ & $0.33 \%$ & $0.34 \%$ & $0.33 \%$ & $0.29 \%$ & \\
\hline & I+D & & & & & & & $0.10 \%$ & $0.11 \%$ & $0.13 \%$ & $0.12 \%$ & $0.11 \%$ & $0.10 \%$ & \\
\hline \multirow{2}{*}{ Uruguay } & ACT & & & & & & & & & & & & & $0.23 \%$ \\
\hline & I+D & $0.25 \%$ & $0.15 \%$ & $0.19 \%$ & $0.07 \%$ & $0.14 \%$ & $0.28 \%$ & $0.28 \%$ & $0.42 \%$ & $0.23 \%$ & $0.26 \%$ & $0.24 \%$ & & $0.22 \%$ \\
\hline Venezuela & ACT & $0.37 \%$ & $0.39 \%$ & $0.49 \%$ & $0.47 \%$ & $0.58 \%$ & $0.61 \%$ & $0.45 \%$ & $0.42 \%$ & $0.37 \%$ & $0.37 \%$ & $0.37 \%$ & $0.48 \%$ & $0.38 \%$ \\
\hline \multirow{2}{*}{\begin{tabular}{|l} 
América \\
Latina y el \\
Caribe
\end{tabular}} & ACT & $0.93 \%$ & $0.93 \%$ & $0.83 \%$ & $0.90 \%$ & $0.90 \%$ & $0.88 \%$ & $0.80 \%$ & $0.73 \%$ & $0.69 \%$ & $0.74 \%$ & $0.77 \%$ & $0.81 \%$ & $0.87 \%$ \\
\hline & I+D & $0.49 \%$ & $0.49 \%$ & $0.43 \%$ & $0.49 \%$ & $0.53 \%$ & $0.58 \%$ & $0.52 \%$ & $0.48 \%$ & $0.45 \%$ & $0.52 \%$ & $0.56 \%$ & $0.59 \%$ & $0.64 \%$ \\
\hline Iberoamérica & I+D & $0.60 \%$ & $0.61 \%$ & $0.59 \%$ & $0.61 \%$ & $0.61 \%$ & $0.64 \%$ & $0.60 \%$ & $0.56 \%$ & $0.56 \%$ & $0.62 \%$ & $0.66 \%$ & $0.69 \%$ & $0.76 \%$ \\
\hline Total & I+D & $2.12 \%$ & $2.16 \%$ & $2.10 \%$ & $2.04 \%$ & $1.96 \%$ & $2.02 \%$ & $2.03 \%$ & $2.05 \%$ & $2.09 \%$ & $2.18 \%$ & $2.24 \%$ & $2.28 \%$ & $2.26 \%$ \\
\hline
\end{tabular}

Fuente: CONCYTEC- Indicadores de Ciencia y Tecnología 1960-2002

Los datos de América Latina y el Caribe son estimados

ACT: Actividades de Ciencia y Tecnología

I+D: Investigación y Desarrollo 


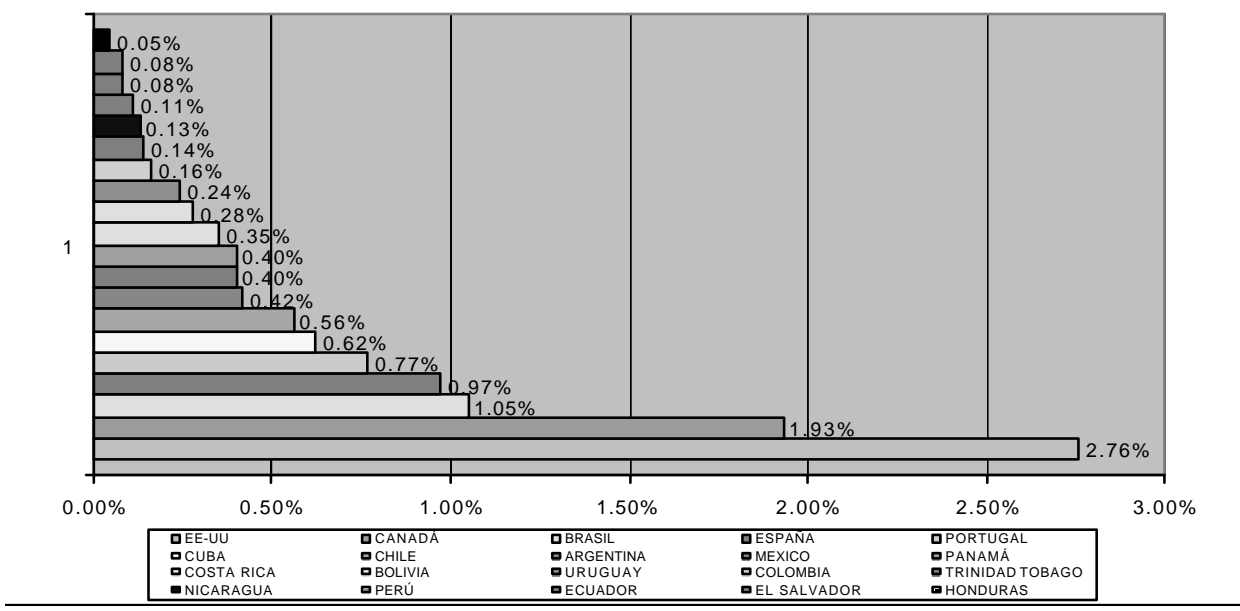

F i g u r a 1. Gastos en inversión y desarrollo como porcentaje del PBI por Países (2001) Fuente: CONCYTEC-Indicadores de Ciencia y Tecnología. 2002

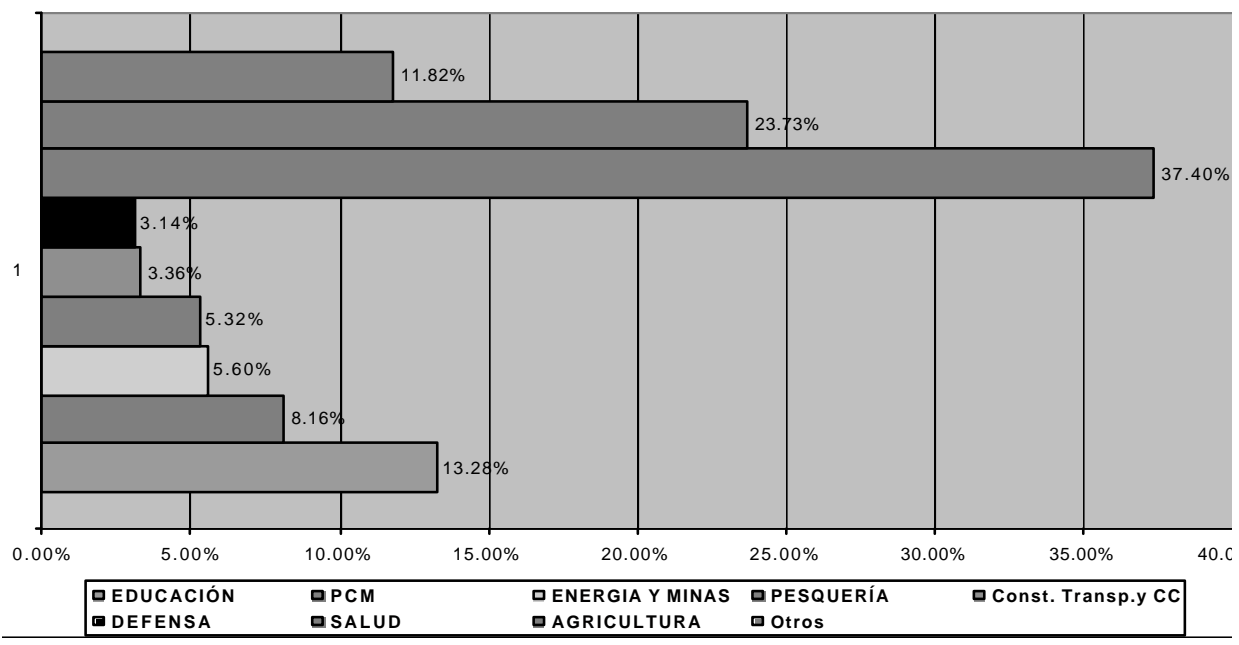

F i g u r a 2. Gasto público en actividades de ciencia y tecnología de Instituciones No Universitarias por sectores 2002 Fuente: CONCYTEC-Indicadores de Ciencia y Tecnología. 2002

Cu a d r o 3. Gastos nacionales en Ciencia y Tecnología por sectores económicos 1998-2002 (en US\$)

\begin{tabular}{|c|c|c|c|c|c|}
\hline \multirow{2}{*}{$\begin{array}{l}\text { SECTORES(Instituciones Públicas } \\
\text { no Universitarias) }\end{array}$} & \multicolumn{5}{|c|}{ AÑOS } \\
\hline & 1998 & 1999 & 2000 & 2001 & 2002 \\
\hline Agricultura & 26009138 & 32380174 & 38836032 & 42072280 & 54708926 \\
\hline Defensa & 8123614 & 6853267 & 7916038 & 7784852 & 7231067 \\
\hline Educación & 23884398 & 25277353 & 30037091 & 34863737 & 30620379 \\
\hline Energía y Minas & 11607943 & 9502448 & 10282692 & 11753839 & 12903387 \\
\hline Presidencia del Consejo de Ministros & 13222140 & 12914777 & 19648495 & 17204596 & 18807254 \\
\hline Pesquería Y Producción & 13639643 & 11326386 & 14910480 & 16384919 & 12903387 \\
\hline Salud & 43398722 & 39807343 & 41758430 & 88873661 & 18807254 \\
\hline Transportes y Comunicaciones & 9284283 & 7354759 & 10795053 & 8416018 & 12268501 \\
\hline Promoción de la Mujer y Desarrollo Humano & 262235 & & & & \\
\hline TOTAL & 149432117 & 145416508 & 174184313 & 227353902 & 230524782 \\
\hline
\end{tabular}

Fuente: Perú ante la Sociedad del Conocimiento, Indicadores de ciencia, Tecnología e Innovación, 1960-2002- CONCYTEC 


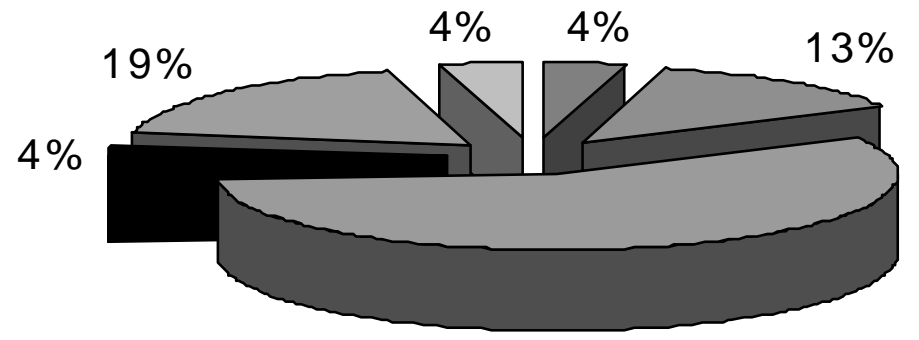

$56 \%$

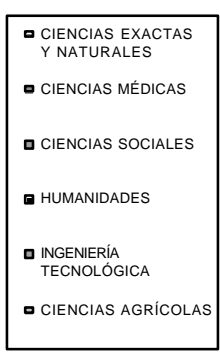

F i g u r a 3. Educación Superior Universitaria: Graduados-Clasificación Unesco, Campos de la Ciencia y Tecnología-2000 Fuente: CONCYTEC-Indicadores de Ciencia y Tecnología. 2002
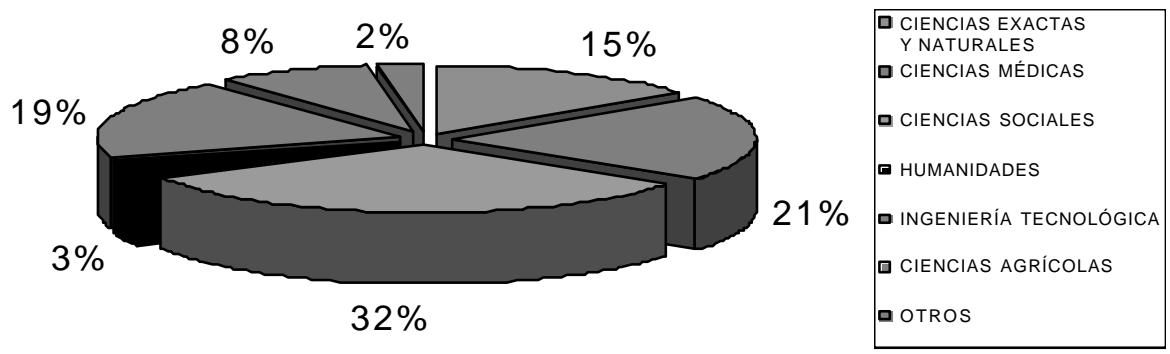

F i g u r a 4. Instituciones de Ciencia y Tecnología según campos de la ciencia y tecnología de la UNESCO 2002 Fuente: CONCYTEC-Indicadores de Ciencia y Tecnología. 2002

ficas o el uso más intensivo y racional de los recursos humanos, técnicos y materiales disponibles. Se observa en la Figura 3, sobre la clasificación de la Ciencia y Tecnología según la UNESCO, con la que se corrobora el comportamiento irracional en nuestro País.

En la Figura 4, se observa el número de instituciones dedicadas a la ciencia y la tecnología, nos da una percepción generalizada de la importancia de la ciencia y la tecnología para el desarrollo y para la solución de problemas de orden social y productivo por parte del Estado, la misma que conlleva al descuido de su importante rol articulador, organizador y catalizador de los esfuerzos nacionales en materia de ciencia y tecnología.

Las paradojas más grandes que tiene el Perú con sus tres regiones: costa, sierra y selva y la cuarta que es el mar ricos en recursos naturales y el tipo de orientación de investigación científica y tecnológica que se imparte, es justamente el tipo de contradicciones que son características de nuestra nación; eso indica que el problema del Perú es de organización.

Ergo, no es un problema de riqueza, ni de capacidad de recursos humanos. Se tiene una discapacidad en la organización como sociedad. No se sabe organizar, no se tiene planes generales, ni nacionales para la agricultura, la minería, la ciencia y tecnología, el turismo; no se tiene nada y así se quiere ser un país con futuro hacia el desarrollo sostenido y sustentable.

PRINCIPALES CAUSAS QUE IMPIDEN EL ORDENAMIENTO INTEGRADO DE LA INVESTIGACIÓN CIENTÍFICA Y TECNOLÓGICA EN EL PERÚ

No se cuenta con un Marco legal único para la promoción de la ciencia y tecnología, lo que se tiene son diferentes leyes de creación de diferentes instituciones del Estado que les asigna funciones que en 
algunos casos hasta se duplican, esta desorganización y desintegración no permite definir las funciones sin caer en duplicidad de las diferentes instituciones públicas y privadas para el desarrollo de la ciencia y tecnología.

No están definidas las áreas de especial interés nacional, que promueva en forma armónica y conjunta el desarrollo humano y la competitividad en el ámbito nacional e internacional.

El Estado no tiene una Política Nacional de Ciencia y Tecnología y menos una Institución rectora que oriente y promueva las actividades de las diferentes instituciones públicas para el desarrollo de la ciencia y tecnología en relación a las necesidades del País.

El no tener un ordenamiento institucional adecuado, está permitiendo que las entidades públicas dedicadas a la ciencia, tecnología e innovación se aparten cada vez mas de la universidad y de la comunidad científica y de las empresas.

Los mecanismos de financiamiento por parte del empresariado no es debidamente estimulado y los fondos provenientes de la Cooperación Técnica Internacional no están siendo utilizados debidamente, comprometiendo a un atraso progresivo en materia de innovación para la competitividad.

Debido a la ausencia de incentivos y soporte institucional adecuados se ha generado un ambiente de incertidumbre en las actividades de investigación científica y tecnológica, en las que el riesgo es cada vez mayor y no cuenta con el respaldo político y económico necesarios. En consecuencia no se propicia $y$, por el contrario, se desincentiva la innovación e investigación por parte del sector privado; se desanima la opción escolar, académica o profesional por las ciencias y la investigación, derivando en desaprovechamiento y fuga de talentos y en incipiente generación de conocimientos e innovación.

Los principales centros de investigación científica y tecnológica reposan sobre el esfuerzo estatal, el cual es insuficiente. Las instituciones públicas invierten muy poco en Investigación y Desarrollo teniendo un marcado sesgo sectorial, que no ha permitido articular adecuadamente la investigación y los servicios especializados de ciencia y tecnología del País, especialmente para propuestas de carácter multisectorial y multidisciplinario que atiendan los requerimientos propios de los principales problemas del desarrollo nacional.

Se ha detenido el incremento de las universidades públicas, mientras que las universidades privadas aumentan con mayor rapidez, sin embargo en ambos casos no están preparadas para la investigación, ya sea por problemas de asignación de recursos o de concepción y gestión, no han impulsado las actividades de investigación, ni la formación de recursos humanos altamente calificados para la generación y acumulación de conocimiento.

No existe una cultura de emprendimiento en la comunidad universitaria, los investigadores son personas centradas en su trabajo específico y no lo ven dentro de un contexto nacional o de competencia.

La participación del sector privado nacional es mínima en la generación, adquisición y adaptación de productos y servicios científicos y tecnológicos. Las empresas no constituyen, como debieran un factor dinamizador por sus requerimientos para la innovación. La gran y mediana empresa aplican tecnologías "llave en mano", que excluyen las capacidades locales para generar, adaptar o participar en la creación e incorporación de conocimiento. La pequeña empresa, no cuenta con mecanismos adecuados para identificar y canalizar sus necesidades de innovación.

\section{CONCLUSIONES}

Dentro del marco de la reforma del Estado, debe conformarse el Sistema de Investigación Científica y Tecnológica, liderado por un organismo rector responsable de definir la política nacional de ciencia y tecnología y formular el Plan Nacional de Ciencia y Tecnología, así como de dirigir, orientar, coordinar, promover, supervisar y evaluar las actividades de ciencia y tecnología a nivel nacional, regional y local, en función de sus necesidades y recursos existentes.

Los mecanismos de articulación y coordinación con las instituciones públicas y privadas, así como de las universidades (respetando el proceso de la descentralización y la concepción de la autonomía) deben ser claros y de cumplimiento obligatorio en el corto, mediano y largo plazo, una vez aprobados.

Comprometer al sector empresarial de la pequeña y mediana empresa, para un trabajo coordinado que garantice la adaptabilidad de los conocimientos de la investigación tecnológica a favor de la innovación para mejorar su competitividad productiva, ya que este es un sector prioritario en la economía del País.

Los incentivos y estímulos a los investigadores deben ser otorgados a los investigadores que cumplan con las orientaciones emanadas por el ente 
rector y cuyos resultados sean de aplicabilidad para solucionar los problemas socioeconómicos de nuestro País.

El establecimiento de una red nacional de información científica y tecnológica de fácil acceso a todos los involucrados en la materia y que sirva como base de información a fin de evitar repeticiones en las investigaciones e inventos.

Administrar el financiamiento y distribución del gasto en ciencia y tecnología en relación a los Planes Nacionales y Regionales a ser formulados por el ente rector.

Finalmente se hace imperativo que se de una ley de creación de un Sistema de Ciencia y Tecnología para ordenar todas las instituciones Públicas y sobre todo definir la Política Nacional de Ciencia y Tecnología. El mencionado sistema debe estar compuesto por las Instituciones Públicas que actualmente realicen estas tareas, las Universidades públicas y privadas y las demás instituciones del ámbito regional y local de manera obligatoria bajo mandato legal. En tanto las entidades privadas u ONGs, fundaciones, empresas u otras deben cumplir con las prioridades emanadas por el ente rector para su debido funcionamiento o financiamiento externo.

\section{BIBLIOGRAFÍA}

1. Concytec. (2002). Perú ante la Sociedad del Conocimiento, Indicadores de Ciencia y Tecnología e innovación, 1960-2002. Lima Perú.

2. Comisión de Educación, Ciencia, Tecnología y Patrimonio Cultural, Congreso de la República del Perú. (2004). Documentos y proyectos de Leyes. Lima Perú.

3. ITDG (2004). Situación de la Ciencia y Tecnología en el Perú y en la Región Latinoamericana. Revista Latinoamericana Tecnología y Sociedad, En: www.itdg.org.pe

4. Mc Lauchlan de Arregui, Patricia; Torero, Máximo. (1998). Indicadores de Ciencia y Tecnología en América Latina. GRADE, Mosca Azul. Lima Perú.

5. Sagasti, Francisco. (1998). Conocimiento y Desarrollo: Ensayos sobre Ciencia y Tecnología. GRADE, Editorial Mosca Azul. Lima Perú.

6. Saravia, Miguel; Marcona, Sandro; Peague, Walter A. (2002). Nuevas Tecnologías ¿Qué nos espera a los Países en Desarrollo?. Editorial ITDG. Lima Perú. 